\title{
Beam transformations by active GRIN materials
}

\author{
C. Gomez-Reino*a, A. I. Gomez-Varela ${ }^{\mathrm{a}}$, M. T. Flores-Arias ${ }^{\mathrm{a}}$, C. Bao-Varela ${ }^{\mathrm{a}}$ and X. de la Fuente ${ }^{\mathrm{b}}$ \\ aGrupo de "Microóptica y Óptica GRIN", Unidad Asociada al Instituto de Ciencias de Materiales de \\ Aragón, ICMA/CSIC, Zaragoza and Escola de Óptica e Optometría, Campus Vida s/n, Universidade \\ de Santiago, E15782 Santiago de Compostela, Spain. \\ ${ }^{\mathrm{b}}$ Instituto de Ciencia de Materiales de Aragón (Universidad de Zaragoza-CSIC), María de Luna 3, \\ E50018 Zaragoza, Spain.
}

INVITED PAPER

\begin{abstract}
Beam transformations process by active materials allows, in a controlled manner, the redistribution of the irradiance and phase of light. A material that exhibits quadratic gain or loss and refractive index profiles is regarded as active GRIN (GRadient-INdex) material. Complex refractive index is examined in order to discuss what loss or gain means in terms of the refractive index. General conditions for beam transformations by active GRIN materials are obtained in a 2D space of the complex curvature. Irradiance evolution through an active selfoc microlens is presented and it is analyzed at planes where beam shaping occurs.
\end{abstract}

Keywords: active material, GRIN optics, Gaussian beams, beam transformations, beam shaping, selfoc microlens.

\section{INTRODUCTION}

Beam transformation is the process of redistributing the irradiance and the phase of a beam optical radiation and a complex material is an optical material that provides gain or loss to an input light beam. A material that has quadratic gain or loss and refractive index profiles in transverse planes away from the optical axis can be regarded as active GRIN material and light propagation through this material has been widely analyzed in optics ${ }^{1-8}$. There are two main purposes in the present paper: to describe general conditions for obtaining no extra-cavity beam transformation by active GRIN materials and to design beam transformers for microoptics. Expression for the complex refractive index is examined in order to discuss what loss or gain means in terms of the index. The formalism of laser beam propagation in GRIN materials regarding as beam transformers with a Gaussian mask is presented. General condition for beam transformations by active GRIN materials as a geometrical solution in 2D space of the complex curvature of the input laser beam is obtained. At the end, results and conclusions concerning to gain and refraction index profiles, transverse and axial irradiances in active GRIN materials are discussed and commented. The obtained results show the important role that can be played by active GRIN microlenses in beam transformations and they are interesting for researchers devoted to material processing by laser, biophotonics and beam homogenizers manufacturing.

\section{LIGHT PROPAGATION IN ACTIVE GRIN MATERIALS: COMPLEX REFRACTIVE INDEX AND BEAM PARAMETERS}

We consider an active GRIN material, with rotational symmetry around the z-axis, of thickness d limited by plane parallel faces whose refractive index is given by a parabolic transverse gain or loss profile

$$
\mathrm{n}(\mathrm{r}, \mathrm{z})=\mathrm{n}_{0}\left[1-\frac{\mathrm{g}^{2}(\mathrm{z})}{2} \mathrm{r}^{2}\right] \text { for } \mathrm{r}=\left(\mathrm{x}^{2}+\mathrm{y}^{2}\right)^{1 / 2} \leq \mathrm{a} \text { and } 0 \leq \mathrm{z} \leq \mathrm{d}
$$

where $\mathrm{n}_{0}$ and $\mathrm{g}(\mathrm{z})$ are the complex refractive index along the $\mathrm{z}$-axis and the complex gradient parameter, respectively.

We assume that $\mathrm{n}_{0}$ and $\mathrm{g}(\mathrm{z})$ can be expressed, by their real and imaginary parts, as

*carlos.gomez-reino@usc.es; phone (+34) 881813512

International Conference on Applications of Optics and Photonics, edited by Manuel F. M. Costa, Proc. of SPIE Vol. 8001, 80011G · C 2011 SPIE · CCC code: 0277-786X/11/\$18 · doi: 10.1117/12.891899 


$$
\begin{gathered}
\mathrm{n}_{0}=\mathrm{n}_{0 \mathrm{R}}+\mathrm{in}_{0 \mathrm{I}} \\
\mathrm{g}(\mathrm{z})=\mathrm{g}_{\mathrm{R}}(\mathrm{z})+\mathrm{ig}_{\mathrm{I}}(\mathrm{z})
\end{gathered}
$$

with $\mathrm{n}_{0 \mathrm{R}}$ and $\mathrm{n}_{0 \mathrm{I}}$ being real constants and $\mathrm{g}_{\mathrm{R}}$ and $\mathrm{g}_{\mathrm{I}}$ real functions of $\mathrm{z}$.

From Eq. (2-3), the real $n_{R}$ and the imaginary $n_{I}$ parts of the complex refractive index, denoted by $\operatorname{Re}[\ldots]$ and $\operatorname{Im}[\ldots]$, are given by

$$
\begin{gathered}
\mathrm{n}_{\mathrm{R}}=\operatorname{Re}[\mathrm{n}(\mathrm{r}, \mathrm{z})]=\mathrm{n}_{0 \mathrm{R}}-\frac{\mathrm{r}^{2}}{2} \operatorname{Re}\left[\mathrm{n}_{0} \mathrm{~g}^{2}(\mathrm{z})\right] \\
\mathrm{n}_{\mathrm{I}}=\operatorname{Im}[\mathrm{n}(\mathrm{r}, \mathrm{z})]=\mathrm{n}_{0 \mathrm{I}}-\frac{\mathrm{r}^{2}}{2} \operatorname{Im}\left[\mathrm{n}_{0} \mathrm{~g}^{2}(\mathrm{z})\right]
\end{gathered}
$$

Using Eq. (4-5), active GRIN materials with gain or loss can be classified ${ }^{9}$. The real part of the refractive index determines the guidance behaviour of the active material in such a way that if $\operatorname{Re}\left[\mathrm{n}_{0} \mathrm{~g}^{2}(\mathrm{z})\right]>0$, $\mathrm{n}_{\mathrm{R}}$ decreases as radial distance increases and the active material has a normal guidance behaviour; on the contrary, if $\operatorname{Re}\left[\mathrm{n}_{0} \mathrm{~g}^{2}(\mathrm{z})\right]<0, \mathrm{n}_{\mathrm{R}}$ increases with increasing radial distance and an anomalous guidance occurs. On the other hand, gain or loss in active GRIN material is determined by the sign of the imaginary part of the refractive index. The material has loss if $n_{I}>0$ and it experiences gain if $\mathrm{n}_{\mathrm{I}}<0$. For on-axis loss $\left(\mathrm{n}_{0 \mathrm{I}}>0\right)$ and $\operatorname{Im}\left[\mathrm{n}_{0} \mathrm{~g}^{2}(\mathrm{z})\right]>0$, loss decreases as distance from axis increases, turning to gain at a given transverse distance. This behaviour is typical for unstable light propagation. If $n_{0 I}>0$ and $\operatorname{Im}\left[\mathrm{n}_{0} \mathrm{~g}^{2}(\mathrm{z})\right]<0$, loss increases with increasing radial distance and this means that stable light propagation occurs. For onaxis gain $\left(\mathrm{n}_{0 \mathrm{I}}<0\right)$ and $\operatorname{Im}\left[\mathrm{n}_{0} \mathrm{~g}^{2}(\mathrm{z})\right]>0$, gain increases along the transverse direction and unstable light propagation is obtained. If $\mathrm{n}_{0 \mathrm{I}}<0$ and $\operatorname{Im}\left[\mathrm{n}_{0} \mathrm{~g}^{2}(\mathrm{z})\right]<0$, gain decreases along the transverse direction and turns to loss for a given radial distance. This is the stable case. Likewise, the real part $\mathrm{g}_{0 \mathrm{R}}$ of gradient parameter determines the transverse parabolic profile and the imaginary part $\mathrm{g}_{0 \mathrm{I}}$ is related to the effect of gain or loss on this parabolic profile.

We suppose now that an active GRIN material is illuminated by a non-uniform monochromatic wave of wavelength $\lambda$ described by a Gaussian beam. We analyze how this Gaussian beam is propagated through the active material in order to characterize the beam parameters. The complex amplitude distribution produced by a spherical Gaussian beam on the input plane of the active GRIN material can be written, apart from complex constant, in the paraxial region by ${ }^{10-12}$

$$
\psi\left(\mathrm{x}_{0}, \mathrm{y}_{0} ; 0\right)=\frac{\mathrm{w}_{0}}{\mathrm{w}(0)} \exp \left\{\mathrm{i} \frac{\pi \mathrm{U}(0)}{\lambda}\left(\mathrm{x}_{0}^{2}+\mathrm{y}_{0}^{2}\right)\right\}
$$

where the complex curvature on the input plane at a distance $\mathrm{d}_{1}$ from the beam waist of diameter $2 \mathrm{w}_{0}$ is given by (Figure 1)

$$
\mathrm{U}(0)=\mathrm{U}_{\mathrm{R}}(0)+\mathrm{iU}_{\mathrm{I}}(0)=\frac{1}{\mathrm{R}(0)}+\mathrm{i} \frac{\lambda}{\pi \mathrm{W}^{2}(0)}=\mathrm{n}_{0} \dot{\mathrm{q}}(0) \mathrm{q}^{-1}(0)
$$

$U_{R}$ and $U_{I}$ being the real and imaginary parts of $U . R(0)$ and $w(0)$ are the radius of curvature and the beam half-width at $\mathrm{z}=0$, respectively, and $\mathrm{q}(0)$ and $\dot{\mathrm{q}}(0)$ are the position and slope of the complex ray at the input plane and where dot denotes derivative with respect to $\mathrm{z}$ evaluated at $\mathrm{z}=0$. The complex ray describes the Gaussian beam propagation through the active material.

The complex curvature at $z_{1}$ is also expressed by

$$
\mathrm{U}\left(\mathrm{z}_{1}\right)=\mathrm{U}_{\mathrm{R}}\left(\mathrm{z}_{1}\right)+\mathrm{i} \mathrm{U}_{\mathrm{I}}\left(\mathrm{z}_{1}\right)=\frac{1}{\mathrm{R}\left(\mathrm{z}_{1}\right)}+\mathrm{i} \frac{\lambda}{\pi \mathrm{w}^{2}\left(\mathrm{z}_{1}\right)}=\mathrm{n}_{0} \dot{\mathrm{q}}\left(\mathrm{z}_{1}\right) \mathrm{q}^{-1}\left(\mathrm{z}_{1}\right)
$$

Eq. (8) can be used for evaluating beam parameters at $z_{1}$, which are given by

$$
\begin{aligned}
\mathrm{R}\left(\mathrm{z}_{1}\right) & =\frac{\left|\mathrm{q}\left(\mathrm{z}_{1}\right)\right|^{2}}{\operatorname{Re}\left[\mathrm{n}_{0} \dot{\mathrm{q}}\left(\mathrm{z}_{1}\right) \mathrm{q}^{*}\left(\mathrm{z}_{1}\right)\right]} \\
\mathrm{w}^{2}\left(\mathrm{z}_{1}\right) & =\frac{\lambda\left|\mathrm{q}\left(\mathrm{z}_{1}\right)\right|^{2}}{\pi \operatorname{Im}\left[\mathrm{n}_{0} \dot{\mathrm{q}}\left(\mathrm{z}_{1}\right) \mathrm{q}^{*}\left(\mathrm{z}_{1}\right)\right]}
\end{aligned}
$$

The irradiance at $z_{1}$ is expressed as 


$$
\mathrm{I}\left(\mathrm{x}_{1}, \mathrm{y}_{1} ; \mathrm{z}_{1}\right)=\left|\psi\left(\mathrm{x}_{1}, \mathrm{y}_{1} ; \mathrm{z}_{1}\right)\right|^{2}=\mathrm{I}_{0} \frac{\exp \left(-2 \mathrm{kn}_{0 \mathrm{I}} \mathrm{z}_{1}\right)}{\left|\alpha\left(\mathrm{z}_{1}\right)\right|^{2}} \exp \left\{-\frac{2\left(\mathrm{x}_{1}^{2}+\mathrm{y}_{1}^{2}\right)}{\mathrm{w}^{2}\left(\mathrm{z}_{1}\right)}\right\}
$$

where $\mathrm{I}_{0}=\mathrm{w}_{0}{ }^{2} / \mathrm{w}^{2}(0)$ and $\alpha\left(\mathrm{z}_{1}\right)=\mathrm{H}_{\mathrm{f}}\left(\mathrm{z}_{1}\right)+\mathrm{U}(0) \mathrm{H}_{\mathrm{a}}\left(\mathrm{z}_{1}\right) / \mathrm{n}_{0}$. A Gaussian irradiance profile is obtained at $\mathrm{z}_{1}$ with on-axis loss or gain determined by the sign of the imaginary part of the refractive index along $\mathrm{z}$ axis. Depending on the sign of the denominator in Eq. (10) $\mathrm{w}^{2}$ can be either positive or negative. If $\mathrm{w}^{2}$ is positive a Gaussian beam is achieved whose irradiance, Eq. (11), is a maximum at axis and decreases with radial distance. If $\mathrm{w}^{2}$ is negative a Gaussian beam is obtained whose irradiance is a minimum along the z-axis and increases with radial distance. Light concentration or light spread around the $\mathrm{z}$-axis occurs as $\mathrm{w}^{2}$ is positive or negative, respectively.

\section{BEAM TRANSFORMATIONS}

There are mainly four functions which microoptic exerts in the various applications: collimation of light, focusing of light, beam shaping and imaging. We will treat on these functions obtained as beam transformations by an active GRIN material. From Eq. (10) it follows that, in general, an input Gaussian beam is converted to Gaussian one by the active GRIN material working as a beam transformer with a Gaussian mask. Transformation of an input Gaussian beam into a uniform distribution at the output can be obtained if the denominator in Eq. (10) is equal to zero. The condition for beam shaping by active GRIN material of thickness $d$ can be written as

$$
\operatorname{Im}\left[\mathrm{n}_{0} \dot{\mathrm{q}}(\mathrm{d}) \mathrm{q} *(\mathrm{~d})\right]=0 \Rightarrow \mathrm{w}(\mathrm{d}) \rightarrow \infty
$$

that is, $w$ at the output of the active material tends to infinite. The active medium is regarded as a beam shaper system: a spherical Gaussian beam is converted to a uniform divergent or convergent spherical wavefront as the curvature radius $R(d)$ is negative or positive, respectively (Figure1). From simple calculation, Eq. (12) can be rewritten as

$$
\left(\mathrm{U}_{\mathrm{R}}(0)-\mathrm{C}_{\mathrm{R}}\right)^{2}+\left(\mathrm{U}_{\mathrm{I}}(0)-\mathrm{C}_{\mathrm{I}}\right)^{2}=\mathrm{h}^{2}
$$

Eq. (13) represents a circumference in $2 \mathrm{D}$ space with cartesian axes the real and the imaginary parts of the complex curvature of the Gaussian beam at the input plane, where $h$ and $C\left(C_{R}, C_{I}\right)$ are the radius and the center of the circumference.

Furthermore, for the beam shaping condition, the complex amplitude distribution at the output becomes

$$
\psi\left(\mathrm{x}_{1}, \mathrm{y}_{1} ; \mathrm{d}\right)=\frac{\mathrm{w}_{0} \mathrm{e}^{-\mathrm{kn}_{01} \mathrm{~d}} \mathrm{e}^{\mathrm{ikn} \mathrm{OR}_{\mathrm{R}} \mathrm{d}}}{|\mathrm{q}(\mathrm{d})|} \exp \left\{-\mathrm{itan}^{-1}\left\{\frac{\operatorname{Im}[\alpha(\mathrm{d})]}{\operatorname{Re}[\alpha(\mathrm{d})]}\right\}\right\} \exp \left\{\mathrm{i} \frac{\pi}{\lambda \mathrm{R}(\mathrm{d})}\left(\mathrm{x}_{1}^{2}+\mathrm{y}_{1}^{2}\right)\right\}
$$

Apart from complex constant factors, Eq. (14) represents a uniform spherical wave of curvature radius

$$
\mathrm{R}(\mathrm{d})=\frac{|\mathrm{q}(\mathrm{d})|^{2}}{\operatorname{Re}\left[\mathrm{n}_{0} \dot{\mathrm{q}}(\mathrm{d}) \mathrm{q} *(\mathrm{~d})\right]}
$$

$R(d)$ is positive if $\operatorname{Re}\left[\mathrm{n}_{0} \dot{\mathrm{q}}(\mathrm{d}) \mathrm{q} *(\mathrm{~d})\right]>0$ (converging wave) and $\mathrm{R}(\mathrm{d})$ is negative if $\operatorname{Re}\left[\mathrm{n}_{0} \dot{\mathrm{q}}(\mathrm{d}) \mathrm{q} *(\mathrm{~d})\right]<0$ (diverging wave).
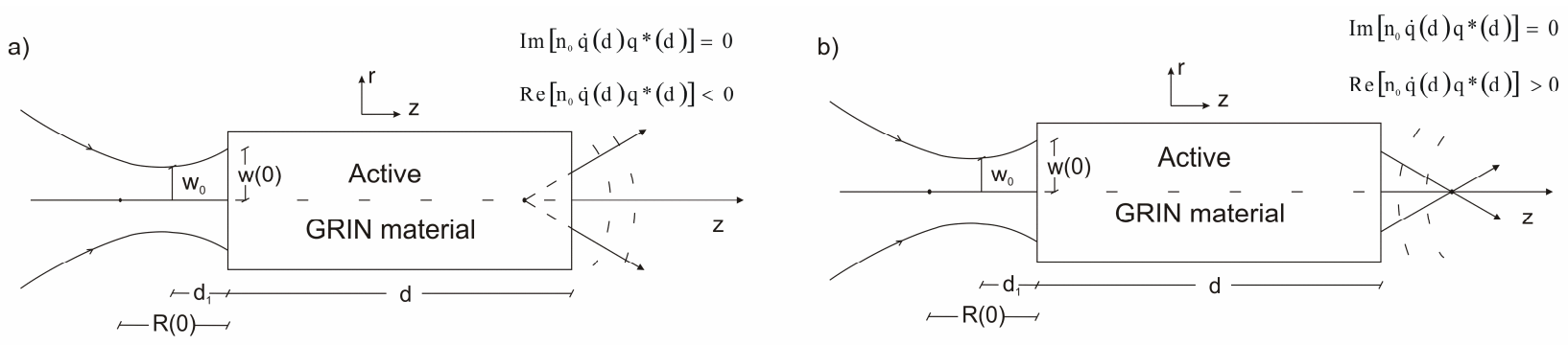

Figure 1. Transformation of a spherical Gaussian beam into a uniform spherical wavefront into a) diverging wavefront $(\mathrm{R}(\mathrm{d})<0)$ and $\mathrm{b})$ converging wavefront $(\mathrm{R}(\mathrm{d})>0)$. 
Another task in beam transformation by active GRIN materials is the conversion of a spherical Gaussian beam to a plane Gaussian beam. The condition for obtaining plane Gaussian beam is expressed as

$$
\operatorname{Re}\left[\mathrm{n}_{0} \dot{\mathrm{q}}(\mathrm{d}) \mathrm{q} *(\mathrm{~d})\right]=0 \Rightarrow \mathrm{R}(\mathrm{d}) \rightarrow \infty
$$

that is, the curvature radius of the Gaussian beam at the output tends to infinite.

We must now connect the complex curvature of the input beam with the complex refractive index of the active GRIN material and the complex axial and field ray. To do this, we rewrite Eq. (16) as follows

$$
\left(\mathrm{U}_{\mathrm{R}}(0)-\mathrm{C}_{\mathrm{R}}^{\prime}\right)^{2}+\left(\mathrm{U}_{\mathrm{I}}(0)-\mathrm{C}_{\mathrm{I}}^{\prime}\right)^{2}=\mathrm{h}^{\prime 2}
$$

To obtain a Gaussian plane beam at the output of the active GRIN material, real and imaginary parts of the complex curvature at the input must be solutions of the circumference given in Eq. (17). $h^{\prime}$ and $C^{\prime}\left(C_{R}^{\prime}, C_{I}^{\prime}\right)$ are the radius and the center of the circumference.

At this case, the complex amplitude distribution at the output becomes

$$
\psi\left(\mathrm{x}_{1}, \mathrm{y}_{1} ; \mathrm{d}\right)=\frac{\mathrm{w}_{0} \mathrm{e}^{- \text {-knord }} \mathrm{e}^{\text {iknord }}}{|\mathrm{q}(\mathrm{d})|} \exp \left\{-\operatorname{itan}^{-1}\left\{\frac{\operatorname{Im}[\alpha(\mathrm{d})]}{\operatorname{Re}[\alpha(\mathrm{d})]}\right\}\right\} \exp \left\{-\frac{\left(\mathrm{x}_{1}^{2}+\mathrm{y}_{1}^{2}\right)}{\mathrm{w}^{2}(\mathrm{~d})}\right\}
$$

Eq. (18) represents a plane Gaussian beam of waist

$$
\mathrm{w}^{2}(\mathrm{~d})=\frac{\lambda|\mathrm{q}(\mathrm{d})|^{2}}{\pi \operatorname{Im}\left[\mathrm{n}_{0} \dot{\mathrm{q}}(\mathrm{d}) \mathrm{q}^{*}(\mathrm{~d})\right]}
$$

Relationships between the real and imaginary parts of the input spherical Gaussian beam that can be converted at the output of a given active GRIN material to a uniform spherical beam or a plane Gaussian beam is mapped as circumferences defined in a 2D space with cartesian axis $\mathrm{U}_{\mathrm{R}}(0)$ and $\mathrm{U}_{\mathrm{I}}(0)$. The circumference always intersects at two points since the following condition is fulfilled providing the limiting cases for which the complex curvature of the output beam tends to zero or infinite. The common solutions of Eq. (13) and (17) are the two points of intersection. One of them corresponds to the case of zero complex curvature at the output, that is

$$
\mathrm{U}(\mathrm{d}) \rightarrow 0 \Rightarrow \mathrm{R}(\mathrm{d}) \text { and } \mathrm{w}(\mathrm{d}) \rightarrow \infty
$$

The input spherical Gaussian beam is transformed into a uniform plane wave at the output of the active GRIN material and a uniform distribution is obtained. The active GRIN material can be regarded as a collimator system.

The complex distribution for zero complex curvature at the output reduces to

$$
\psi\left(\mathrm{x}_{1}, \mathrm{y}_{1} ; \mathrm{d}\right)=\frac{\mathrm{W}_{0} \mathrm{e}^{- \text {knold }} \mathrm{e}^{\text {iknord }}}{|\mathrm{q}(\mathrm{d})|} \exp \left\{-\operatorname{itan}^{-1}\left\{\frac{\operatorname{Im}[\alpha(\mathrm{d})]}{\operatorname{Re}[\alpha(\mathrm{d})]}\right\}\right\}
$$

that is, a uniform plane wave of amplitude

$$
\frac{\mathrm{w}_{0}}{|\mathrm{q}(\mathrm{d})|} \mathrm{e}^{-\mathrm{knold}}
$$

The other point provides the case of infinite complex curvature at the output

$$
\mathrm{U}(\mathrm{d}) \rightarrow \infty \Rightarrow \mathrm{R}(\mathrm{d}) \text { and } \mathrm{w}(\mathrm{d}) \rightarrow 0
$$

The input spherical Gaussian beam is converted to a point source at the output and the active GRIN material works as a focuser system. At this case, the complex amplitude distribution at the output becomes 2D delta Dirac function.

\section{RESULTS AND DISCUSSION}

To illustrate beam shaping by an active GRIN material and to design an active beam shaper, we apply above results to an active selfoc microlens with on-axis gain whose complex gradient parameter is a constant $\mathrm{g}_{0}$ given by

$$
\mathrm{g}_{0}=\mathrm{g}_{0 \mathrm{R}}+\mathrm{ig}_{0 \mathrm{I}}
$$


Figure 2 depicts the parabolic behaviour of the real and imaginary parts of the complex refractive index for an active selfoc microlens with gain. For this case the values $\mathrm{n}_{01}=-10^{-5}, \mathrm{n}_{\mathrm{I}}(\mathrm{a})=-10^{-4}$ have been considered.
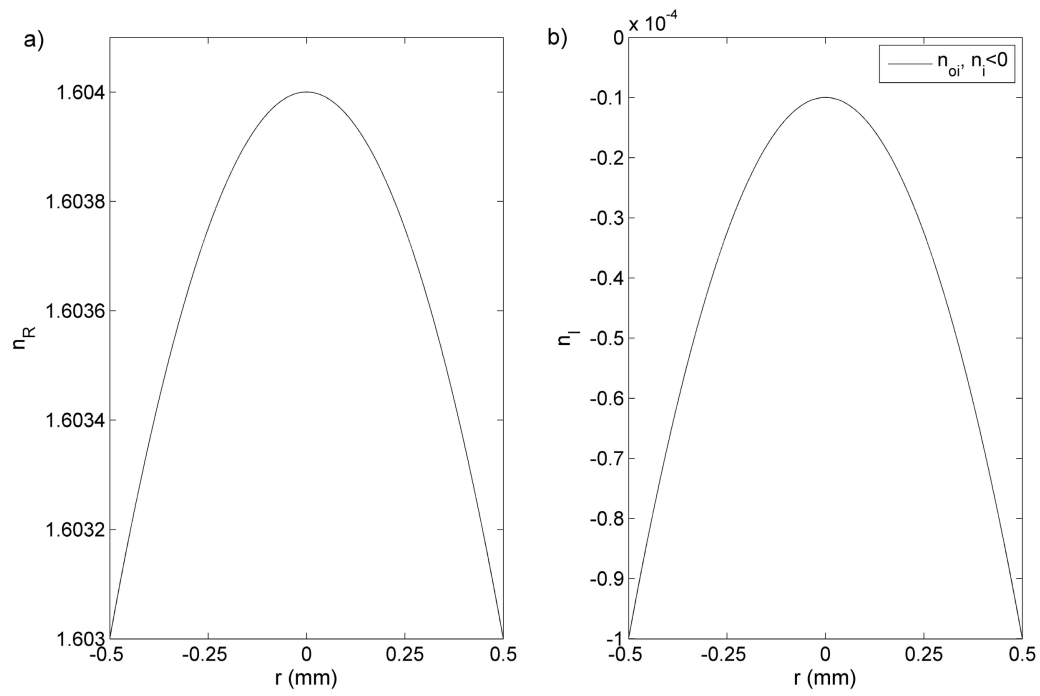

Figure 2. Plotting of a) real and b) imaginary parts of the complex refractive index for an active selfoc microlens of semiaperture $\mathrm{a}=0.5 \mathrm{~mm}, \mathrm{n}_{0 \mathrm{R}}=1.604$ and $\mathrm{n}_{\mathrm{R}}(\mathrm{a})=1.603$ exhibiting on-axis gain. Parameter values: $\mathrm{n}_{0 I}=-10^{-5}, \mathrm{n}_{\mathrm{I}}(\mathrm{a})=-10^{-4}$, $\mathrm{g}_{0 \mathrm{R}}=0.070694 \mathrm{~mm}^{-1}$ and $\mathrm{g}_{0 \mathrm{I}}=0.003175 \mathrm{~mm}^{-1}$.

The real part of the refractive index (Figure 2a) decreases with increasing radial distance so the active microlens presents normal guidance propagation. Figure $2 b$ ) represents the imaginary part of the refractive index. A parabolic behaviour is shown and the values of the imaginary part of the refractive index decreases from the center to the edges of the microlens. The imaginary part of the refractive index is negative and a concentration of energy around the axis occurs.

For simulation the irradiance distribution versus $z_{1}$ for an active selfoc microlens with on-axis gain illuminated by a tunable Ti:Sapphire laser operating at wavelengths 790 and $1050 \mathrm{~nm}$ is depicted in Figure 3. For a distance of $25 \mathrm{~mm}$ from the beam waist to the input face of the active microlens, the beam half-width is bigger for $\lambda=790 \mathrm{~nm}$ than for $\lambda=1050 \mathrm{~nm}$. The irradiance increases with propagation and the beam radius becomes wider (diverging Gaussian beam) until a length for which the beam shaping condition is fulfilled. The Gaussian beam is transformed into a uniform beam (enlarged regions) with constant irradiance at thicknesses of 1.549 and $2.063 \mathrm{~mm}$ for 790 and $1050 \mathrm{~nm}$, respectively. Therefore, uniform beam reaches at the shaping length. For length higher than shaping length the uniform beam is converted again to a Gaussian beam whose irradiance increases with propagation. Relative irradiance increases with penetration in the active media due to on-axis gain effects. As the beam propagates through the active microlens we find that a strong variation of the relative irradiance takes place corresponding to the length where the Gaussian beam changes from convergent to divergent. At thicknesses of $22.135 \mathrm{~mm}$ and 22.138 , for wavelengths of $790 \mathrm{~nm}$ and 1050 $\mathrm{nm}$, the radius of curvature of the beam changes its sign and a concentration of the irradiance occurs. Beam shaping is achieved again at thicknesses of $29.225 \mathrm{~mm}$ for $790 \mathrm{~nm}$ and $28.985 \mathrm{~mm}$ for $1050 \mathrm{~nm}$, obtaining in both cases a uniform beam with a higher irradiance value than for the first beam shaping length due to the gain effects. On the other hand, comparing Figure 3a) and 3b), we can see that higher values of the irradiance are achieved for wavelength of $790 \mathrm{~nm}$ than for $1050 \mathrm{~nm}$.

In Figure 4 the top view of the beam propagation through the active selfoc microlens is shown. A Gaussian beam is launched into an active selfoc microlens with on-axis gain. As it propagates in the active media, it is transformed to a uniform beam at the first thickness where the beam shaping condition is fulfilled. From this point on, the irradiance increases while it concentrates around the axis until a point where it reaches a maximum. Then, the irradiance value decreases with propagation and, when the beam shaping condition is verified again, the Gaussian beam is transformed into a uniform beam. After this, the beam suffers again the transformations described in this paragraph. 
a)

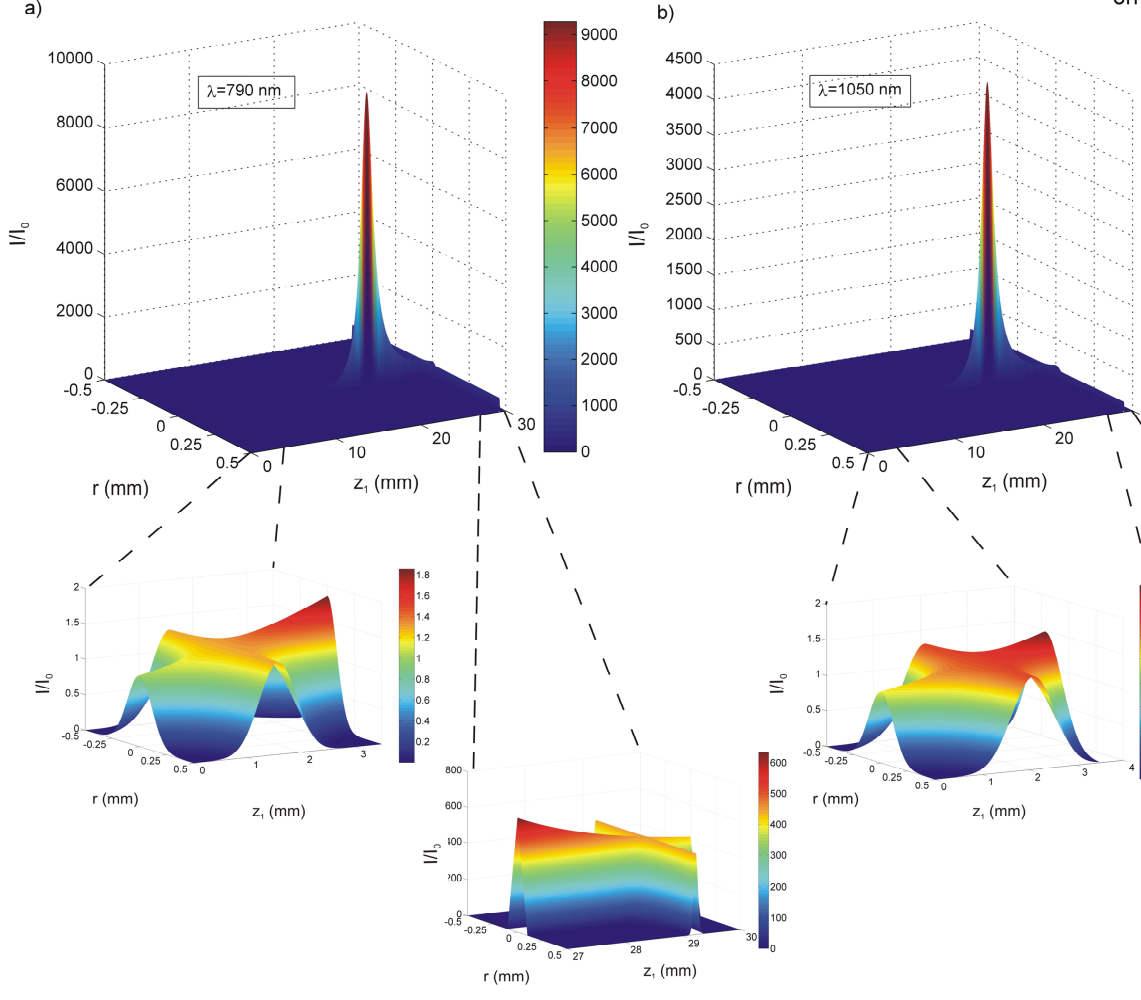

on-axis gain

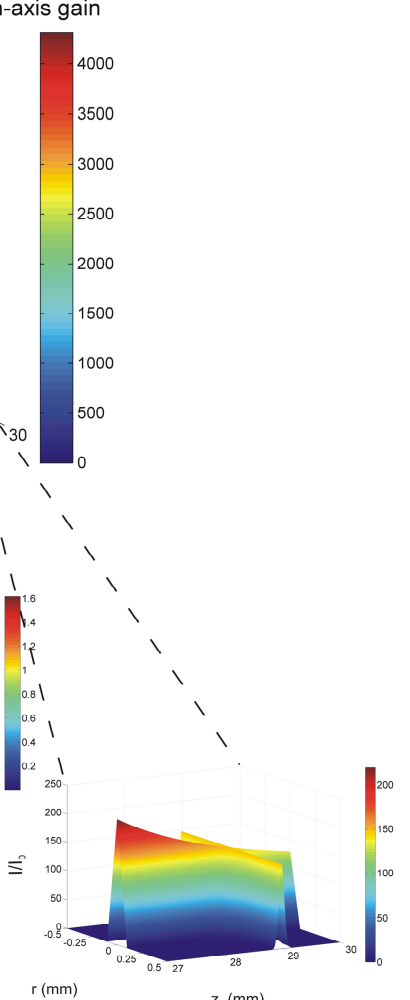

Figure 3. Relative irradiance $I / I_{0}$ versus $z_{1}$ for an active selfoc microlens with on-axis gain illuminated by Ti:Sapphire laser with a) $\lambda=790 \mathrm{~nm}$ and $2 \mathrm{w}_{0}=0.95 \mathrm{~mm}$ and b) $\lambda=1050 \mathrm{~nm}$ and $2 \mathrm{w}_{0}=0.95 \mathrm{~mm}$. For both cases $\mathrm{d}_{1}=25 \mathrm{~mm}$. Calculations have been made for parameter values of Figure 2 .
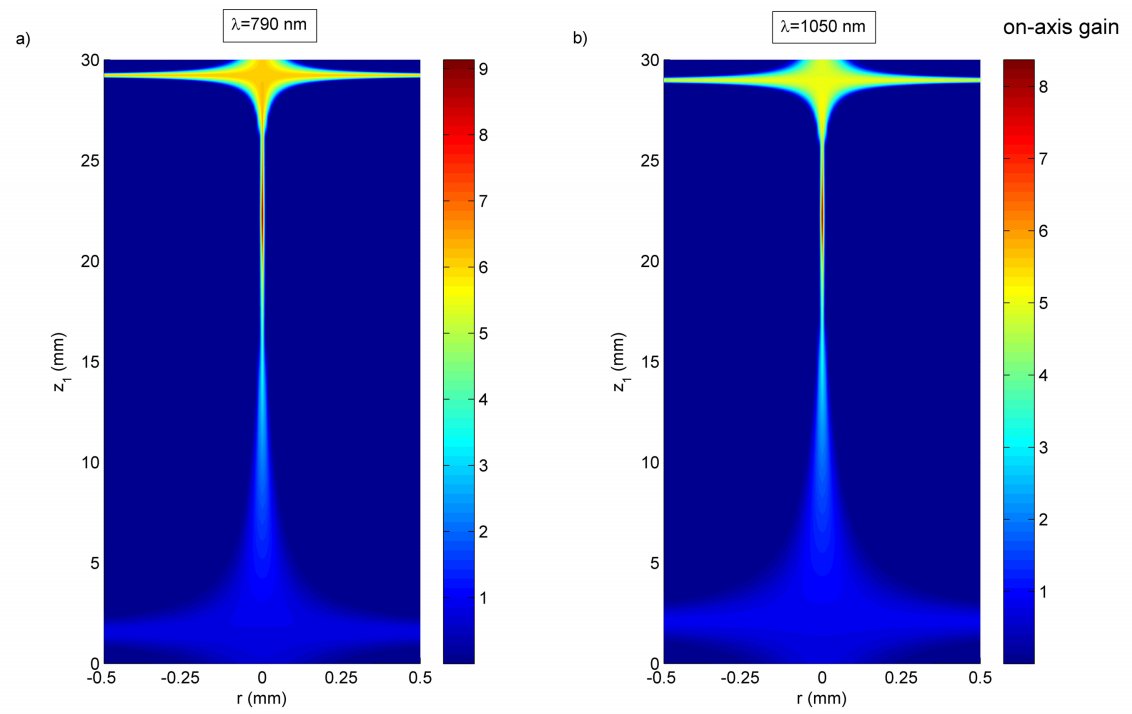

Figure 4. Top view of the beam propagation in the active selfoc microlens with on-axis gain for a) $790 \mathrm{~nm}$ and b) $1050 \mathrm{~nm}$. In both cases beam shaping and concentration of light occurs during the beam propagation in the active media. Irradiance values have been rescaled in order to obtain an appropriate color map in the figure to make easier its interpretation. 
Beam half-width evolution is plotted in Figure 5 for the two wavelengths of the tunable Ti:Sapphire laser. Both present very slight changes in their behaviour except for the two thicknesses where $\mathrm{w}^{2}$ changes its sign, in other words, where the Gaussian beam becomes a uniform beam. $\mathrm{w}^{2}$ is positive below the first shaping length, changes of sign at shaping length, and is negative above shaping length. At $\mathrm{z}$ well below and above shaping length $\mathrm{w}^{2}$ remains almost constant. At shaping length beam half-width reaches its peak value. The same happens for the second thickness where beam shaping occurs, but now $\mathrm{w}^{2}$ is negative below shaping length, changes of sign at shaping length, and is positive above shaping length.
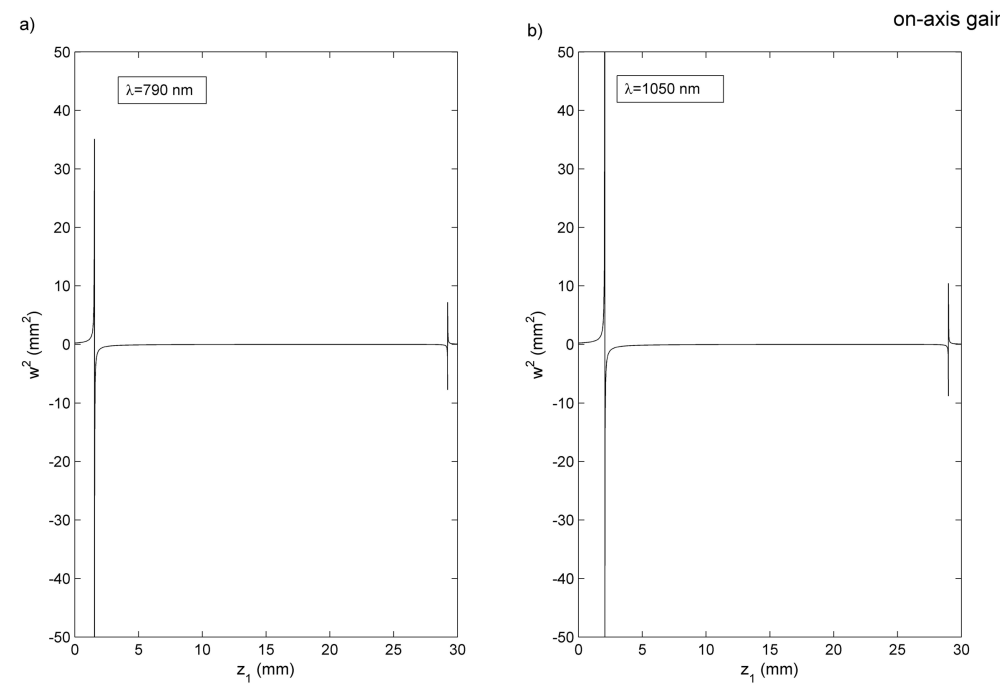

Figure 5. Beam half-width evolution in an active selfoc microlens with on-axis gain. Plotting of the beam half-width versus $z_{1}$ in an active selfoc microlens of thickness $30 \mathrm{~mm}$ for wavelengths a) 790 and b) $1050 \mathrm{~nm}$, respectively. Calculations have been made for parameter values of Figure 2.

Figure 6 shows the behaviour of the radius of curvature of the Gaussian beam with propagation in the active selfoc microlens for on-axis gain case. For both wavelengths the radius of curvature presents a similar behaviour: it increases up to the region where the radius of curvature changes its sign, that is, where the beam converts from convergent to divergent.
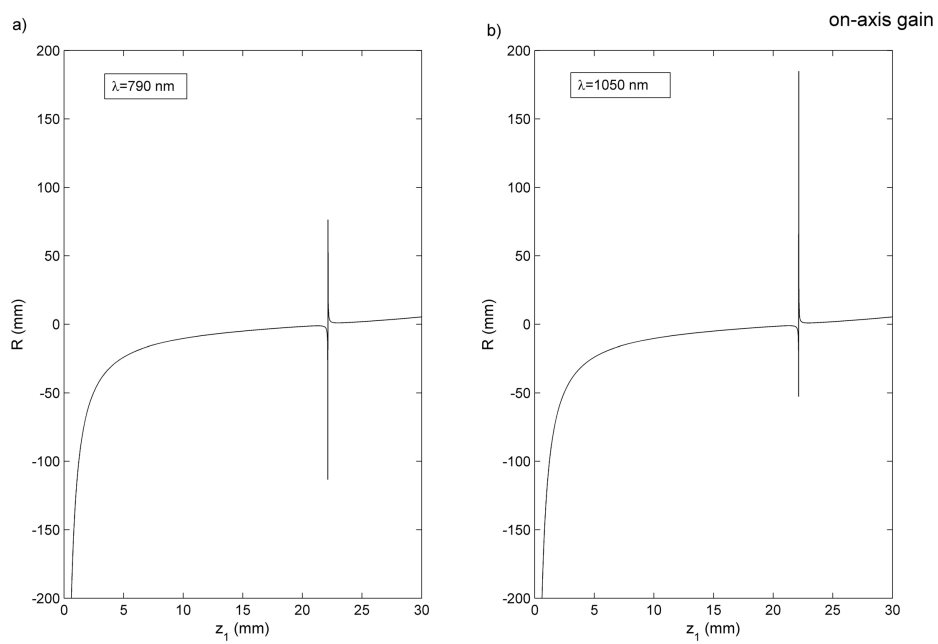

Figure 6. Plotting of the radius of curvature of the Gaussian beam in an active selfoc microlens with on-axis gain versus $\mathrm{z}_{1}$ of thickness $30 \mathrm{~mm}$ for wavelengths a) $790 \mathrm{~nm}$ and b) $1050 \mathrm{~nm}$. Calculations have been made for parameter values of Figure 2 . 


\section{CONCLUSIONS}

We have studied beam transformations performed by active GRIN materials. Light propagation of Gaussian beams through active materials has been analyzed by using complex refractive index and gradient parameter as an analytical extension from expressions for passive materials. Results have been applied to light propagation in active selfoc microlens with on-axis gain in order to show that a uniform irradiance distribution can be obtained from transformation of Gaussian beams. Lengths where the beam shaping condition is fulfilled have been studied and corresponding thicknesses of the active selfoc microlens for beam shaping have been evaluated. The beam half-width evolution of a Gaussian beam propagating in the active microlens with gain has been analyzed finding a change of its sign for thicknesses where uniform beam is achieved. Finally, evolution of the radius of curvature has also been studied resulting that a high energy concentration occurs at thicknesses where it changes its sign, that is, where the Gaussian beam transforms from convergent to divergent.

\section{REFERENCES}

[1] Kogelnik, H., "On the Propagation of Gaussian Beams of Light Through Lenslike Media Including those with a Loss or Gain Variation," Appl. Opt. 4, 1562-1569 (1965).

[2] Casperson, L. W. and Yariv, A., "The Gaussian mode in optical resonators with a radial gain profile," Appl. Phys. Lett. 12, 355-357 (1968).

[3] Casperson, L. W., "Beam modes in complex lenslike media and resonators," J. Opt. Soc. Am. 66, 1373-1379 (1976). [4] Nazarathy, M, Hardy, A. and Shamir, J., "Generalized mode propagation in first-order optical systems with loss or gain,” J. Opt. Soc. Am. 72, 1409-1420 (1982).

[5] Martínez-Herrero, R. and Mejías, P. M., "Beam characterization through active media," Opt. Comm. 85, 162-166 (1991).

[6] Liñares, J., Gomez-Reino, C, Flores, J. R. and Acosta, E., "Focal shift in lenslike media with gain or loss," J. Mod. Opt. 35, 679-691 (1988).

[7] Gomez-Reino, C., Acosta, E., Gonzalez, R. Liñares, J. and Flores, J. R., "Transformation of a gaussian beam into a uniform beam by lenslike medium with gain or loss," J. Mod. Opt. 38, 317-326 (1991).

[8] Gomez-Reino, C., Perez, M. V. and Bao-Varela, C., [Gradient-Index Optics: Fundamentals and Applications], Springer Verlag, Berlin, chap. 5, (2002).

[9] Marcuse, D., [Light transmission optics], 2nd Edition, Van Nostrand Reinhold, cop., New York, chap. 7, (1982).

[10] Arnaud, J., [Beam and Fiber Optics], Academic Press, New York, San Francisco, and London, chap. 2, (1976).

[11] Siegman, A. E., [Lasers], University Science, Mill Valley, California, chap. 15-17, (1986).

[12] Saleh, B. E. A. and Teich, M. C., [Fundamentals of Photonics], John Wiley \& Sons, Hoboken, New Jersey, chap. 3, (2007).

\section{ACKNOWLEDGEMENTS}

This work has been supported by the projects INCITE08PXIB206013PR Xunta/Feder and MAT2010-18519, Ministerio de Ciencia e Innovación, Spain. Besides, Ana I. Gomez-Varela wants to acknowledge the financial support from the FPU (Formación de Profesorado Universitario) grant 2009 (Ministerio de Educación, Spain). 